\section{APC mutants for ALS}

\section{By Lev Osherovich, Senior Writer}

A study by University of Rochester researchers suggests that a modified form of activated protein $\mathrm{C}$ could be used to treat a common form of familial amyotrophic lateral sclerosis. ${ }^{1}$ The findings also suggest that inflammatory activation of microglia could be an early intervention point to stop the disease.

The findings are licensed to ZZ Biotech LLC, which was cofounded in 2008 by study leader Berislav Zlokovic. The company is optimizing the activated protein C (APC) variant to maximize its anti-inflammatory effects and reduce its anticoagulant activity.

APC is a plasma protease that has two main mechanisms. One involves degrading Factor V (F5; FVL) and Factor VIII (F8; AHF), which leads to anticoagulant effects. The other mechanism involves APC binding to protease-activated receptors (PARs), which stimulates anti-inflammatory and neuroprotective pathways.

Xigris drotrecogin alfa, a recombinant form of APC from Eli Lilly and Co., takes advantage of both mechanisms in its approved indication of sepsis.

Zlokovic, a professor of neurology at the University of Rochester Medical Center, has been focused on separating APC's anti-inflammatory activity from the protein's anticoagulant effects to treat a variety of neurological indications. ${ }^{2}$

The new findings by his group, published in The Journal of Clinical Investigation, provide details about how APC's activation of PARs reduces expression of superoxide dismutase 1 (SOD1), a misfolded protein that is responsible for $20 \%$ of familial ALS cases.

Step one was separating out the two main mechanisms of APC. For that, Zlokovic's team turned to collaborators at The Scripps Research Institute. Scripps provided a set of mutant APC variants that had about $70 \%$ lower anticoagulant activity than wild-type protein.

The Rochester researchers systemically injected the APC variants into mice that overexpressed an ALS-linked mutant version of SOD1 and found that APC entered the brain via the spinal cord and slowed ALS progression. APC-treated mice had higher body weight and better survival rates than mock-treated controls.

"We have a biologic-APC-that we can infuse peripherally to modulate the levels of a mutant gene in familial ALS," said Zlokovic.
He added that the APC variants arrested disease progression in mice that "already have the disease and are losing weight."

\section{Micro(glia) management}

APC appears to lower transcription of SOD1 in microglia, which are the brain's innate immune cells. In cultured microglia, APC treatment lowered levels of SOD1 mRNA compared with mock treatment.

Zlokovic suspects that high SOD1 levels stimulate the inflammatory activity of microglia at the very onset of ALS, preceding the weight loss and neurological defects that follow. He noted that knocking out SOD1 exclusively in endothelial cells, which also respond to APC, did not prevent disease progression in mice.

"By specifically deleting SOD1 in various cells, we found that the primary effect of the protein is in microglia," said Zlokovic. "It could be that microglia are the direct cause of the disease."

The team also found that PARs were necessary for the therapeutic effect of APC. Indeed, one particular APC mutant that was unable to activate PARs did not alter ALS progression.

The study shows "a positive effect in the gold-standard animal model of ALS; they have a mechanism by which their protein gets to the desired site across the blood spinal cord barrier-a challenge in this disease-and they have dissected a potential mechanism of action, namely the downregulation of SOD1 expression," said Philip Gregory, CSO and VP of research at Sangamo BioSciences Inc.

Sangamo's SB-509, a zinc-finger protein therapeutic that boosts the transcription of VEGF-A, is in Phase II testing to treat ALS.

Because the anticoagulant effects of APC are unnecessary for efficacy in neurological indications, Zlokovic's team is searching for improved mutants with greater specificity for the PAR-mediated effects of APC. The group also plans to test whether APC mutants could be useful in more common, sporadic cases of ALS that are not associated with SOD1 mutations.

\section{Catching Zs}

Zlokovic's findings are covered by patents issued to the University of Rochester and Scripps, and the IP is licensed to ZZ Biotech. CFO Joe Romano said the company's lead molecule is an APC variant similar to those described in the JCI study.

In 2006, Zlokovic's team found that APC also counteracts the hemorrhagic side effects of thrombolytic therapy for stroke through the same PAR-dependent neuroprotective mechanism seen in the ALS study. ${ }^{3}$

Romano said the company plans to start a Phase I trial of engineered APC variants for stroke in 2010, whereas the ALS program is still in preclinical development.

In September, ZZ Biotech announced a deal with Laureate Pharma Inc., a contract biologics manufacturer, to scale-up APC production to GMP standards in preparation for clinical trials. 


\section{TARGETS \& MECHANISMS}

Osherovich, L. SciBX 2(45); doi:10.1038/scibx.2009.1650

Published online Nov. 19, 2009

\section{REFERENCES}

1. Zhong, Z. et al. J. Clin. Invest.; published online Oct. 19, 2009; doi:10.1172/JCl38476

Contact: Berislav V. Zlokovic, University of Rochester Medical Center, Rochester, N.Y.

e-mail: berislav_zlokovic@urmc.rochester.edu

2. Mosnier, L.O. et al. Blood 109, 3161-3172 (2007)
3. Cheng, T. et al. Nat. Med. 12, 1278-1285 (2006)

COMPANIES AND INSTITUTIONS MENTIONED

Eli Lilly and Co. (NYSE:LLY), Indianapolis, Ind.

Laureate Pharma Inc., Princeton, N.J.

The Scripps Research Institute, La Jolla, Calif.

Sangamo BioSciences Inc. (NASDAQ:SGMO), Richmond, Calif.

University of Rochester, Rochester, N.Y.

University of Rochester Medical Center, Rochester, N.Y.

ZZ Biotech LLC, Rochester, N.Y. 